\title{
Estimación de niveles erosivos en suelos con cultivo de piña en Sarapiquí
}

\author{
Maycol Álvarez Rojas ${ }^{1} \&$ Wagner Peña ${ }^{2}$ \\ 1. TFG MARENA, Escuela de Ciencias Exactas y Naturales, UNED. \\ 2. Cátedra Gestión sostenible del suelo, ECEN, UNED.
}

Recibido: 12 noviembre 2012

Aceptado: 04 febrero 2013

\section{RESUMEN}

La creciente expansión que presentan los sembradíos de piña en Costa Rica, ha levantado preocupaciones, debido a la alta mecanización de los procesos de sembrado que ha ocasionado fuertes niveles de erosión que han saturado de sedimentos ríos, quebradas y hasta importantes humedales como el de Caño Negro en la zona Norte (Programa Estado de la Nación, 2005). Con base a este hecho el trabajo propuesto se extendió sobre el objetivo de medir el grado de erosión y pérdida de suelo en los cultivos de piña, en los terrenos de la empresa Piñales Santa Clara S.A., empleando dos metodologías distintas, la primera de ellas se basó en cuantificar los sedimentos arrastrados por el agua, cuyos datos logrados mostraron una estimación de 49 Ton/ ha/año. Con la segunda metodología se estimó la erosión a través de la medición de pedestales, obteniéndose un valor de 142 Ton/ha/año. Los datos de erosión calculados con ambos métodos se enmarcaron dentro de la categoría de erosión severa, según parámetros del MAG et al. (1994), que establecen que este tipo de erosión abarca los valores de suelo perdido entre 50 y 200 ton/ha/año. Además, el estudio realizado involucró la medición de la capacidad hídrica y la densidad aparente de los suelos, con resultados que mostraron la contribución de estos dos factores al proceso erosivo medido en los terrenos estudiados.

Palabras clave: erosión, piña, suelos.

\footnotetext{
ABSTRAC

The growing expansion posed by pineapple plantations in Costa Rica, has raised concerns due to the high mechanization of processes that caused strong seeded erosion levels have saturated sediment that rivers, streams and wetlands as Caño Negro in the North (Estado de La Nación, 2005). Based on this fact the proposed work is spread over the objective of measuring the degree of erosion and soil loss in pineapple crops on the grounds of the Santa Clara company Piñales SA, using two different methodologies, the first of which is quantified based on sediments carried by water, whose data showed 49 tons/ha/year. With the second methodology was estimated erosion through pedestals
}

measurement, obtaining a value of 142 tons/ha/year. Soil loss data are calculated by both methods framed within the category of severe erosion as parameters MAG et al. (1994), which indicated that covers this type of erosion of soil loss values between $50 \& 200$ ton/ha/year. In addition, the study involved the measurement of the water capacity and the bulk density of the soil, with results showing the contribution of these factors to process the fields measured erosive studied.

Key words: Soil loss, pineapple, soils.

\section{Introducción}

\section{Antecedentes}

Los esfuerzos en conservación de suelos se comenzaron a manifestar en nuestro país a inicios de la segunda mitad del siglo pasado, con acciones como: la formulación de la ley No. 1540 para la creación de un programa de conservación de suelos (MAG et al., 1994; MAG/ FAO, 1997), la entrada en funcionamiento en el año 1985 del proyecto Apoyo al servicio de conservación de suelos y aguas (GCP09/COS/009/ ITA), con el cual se dio la creación del Servicio Nacional de Conservación de Suelos y Aguas (SENACSA) organismo que atendería el problema del deterioro de los suelos en el país (MAG et al., 1994) y que conseguiría en 1991, el apoyo para el proyecto llamado Fomento y aplicación de prácticas de manejo y conservación de tierras en Costa Rica (GCP/COS/012/ NET) (MAG et al., 1994). Además en 1998 se daría la creación de la Comisión Asesora sobre Degradación de Tierras (CADETI), bajo el Decreto ejecutivo 27258-MINAE. Este órgano se encargaría de elaborar instrumentos técnicos 
y científicos de ejecución y asesoría para cumplir con los principios mencionados en la convención realizada por las Naciones Unidas, para combatir la desertificación y la sequía. Un año después CADETI iniciaría su trabajo con la convocatoria a la primera jornada de sensibilización sobre degradación de tierras, para proponer el análisis y discusión de posibles mecanismos de control, mitigación y rehabilitación de las áreas identificadas en el país con deterioro de suelos (CADETI-MINAE, 1999).

En síntesis, los esfuerzos aplicados a nivel nacional para el buen uso, manejo y conservación de suelos, han girado sobre proyectos y decretos que atacan los problemas del sector agropecuario en forma conjunta, sin enfocarse a problemas de manera puntual por cada actividad en específico, y como un caso a resaltar destaca la actividad piñera que a pesar de que su surgimiento alrededor de 1950, coincidió con los primeros esfuerzos pioneros en la conservación del suelo, su aporte al mismo ha sido escaso, hecho que se ve reflejado hoy día, donde a parte del éxito económico que sin duda ha presentado la actividad, la expansión de las fincas piñeras por el país ha repercutido en el deterioro del suelo en cuatro dimensiones que son: erosión, compactación, perdida de actividad microbiológica y producción como mono cultivo (Quijandría y Prats, Citado por Gonzáles, 2005).

\section{El problema}

Dentro de las actividades agrícolas desarrolladas en nuestro país, el cultivo de la piña ha llamado la atención, debido a que sus procesos de producción altamente mecanizados y su expansión por cuencas como Reventazon-Parismina, Sarapiquí, Pocosol, Cureña y el Grande de Térraba (Programa Estado de la Nación, 2005), han causado la saturación de los causes de ríos y quebradas con gran cantidad de sedimentos, transformando a los lugares finales de deposición del suelo vulnerables a inundaciones y otros problemas ambientales. Es por ello que se propone medir el grado de erosión y pérdida de suelo, recurso natural que se supone hay que conservarlo y mejorar sus propiedades. La propuesta implica demostrar la pérdida del suelo, como ejemplo se realizará en los terrenos de la empresa Piñales Santa Clara S.A., Sarapiquí. Cabe indicar que no todas las empresas están asociadas a la Cámara Nacional de Piñeros (CANAPEP) y lo que sí lo están deben cumplir una norma de Gestión Socioambiental.

\section{Materiales y métodos}

La investigación planteada es del tipo experimental - explicativa, por incorporar el desarrollo de dos metodologías para la estimación de la pérdida de suelo dentro de cultivos de piña y la realización de un trabajo de muestreo y análisis de suelo, sobre las propiedades físicas de conductividad hídrica y densidad aparente.

Dicha experimental se realizó en el año 2007, en los terrenos de la empresa Piñales Santa Clara S.A., ubicada en las cercanías del poblado de San Gerardo, perteneciente al distrito La Virgen, del cantón de Sarapiquí, en la provincia de Heredia. La topografía de la zona se caracteriza por ser ondulada, el régimen de precipitación promedio ronda los $4,000 \mathrm{~mm}$ al año y las temperaturas oscilan entre los $20 \mathrm{y}$ $30{ }^{\circ} \mathrm{C}$ (SUNII/MAG, 2006). El Río Sardinal, Quebrada Grande y Quebrada Sonora, son los afluentes que se hayan influenciados por el sitio de estudio (IGNCR, 1985).

Como instrumentos para recolectar la información se emplearon: cilindros metálicos para tomar muestras de suelo, horno para secados de muestras de suelo, sistema de posicionamiento global (GPS), libreta de campo, cámara fotográfica, espátula de albañil, regla de medición, balanza portátil y medios estañones metálicos.

\section{Medición de la densidad aparente}

Para conocer la densidad aparente de los suelos estudiados, se extrajeron 3 muestras de un lote identificados como 7308 y dos muestras del lote numerado como 7311, a una profundidad no mayor a los $30 \mathrm{~cm}$, empleando cilindros metálicos, cuyo peso en gramos y capacidad volumétrica debió ser medida. 
Las muestras extraídas cuidadosamente fueron llevadas a laboratorio, y horneadas por 24 horas a una temperatura de $105^{\circ} \mathrm{c}$, para dejarlas libres de humedad. Por ultimo al peso de la muestra del suelo seco dentro del cilindro, se le restó el peso del cilindro, para conseguir como resultado sólo el valor de la masa del suelo seco, que sería dividida entre el volumen del cilindro, para obtener el valor de densidad aparente.

\section{Determinación del Potencial hídrico del suelo}

En la determinación del potencial hídrico del suelo, se utilizaron cilindros metálicos iguales a los empleados para la densidad aparente, las muestras fueron tomadas a dos profundidades distintas, debido a que en el sitio de la investigación los terrenos se hayan preparados y se podía encontrar diferencias de potencial entre los primero $20 \mathrm{~cm}$ y después de los $20 \mathrm{~cm}$. Las muestras tomadas fueron llevadas al laboratorio, donde se les determinó el área de la base del cilindro, que se denominó valor "A" y la altura, que se llamó valor "L", luego a cada muestra se le sujetó un papel de filtro y cedazo en su base y se adiciono otro cilindro en la parte superior, que conservaría la carga hídrica, la cual debía tener un nivel constante. Posteriormente se midió la altura total de los dos cilindros unidos, y se llamó valor "H". Las muestras fueron dejadas en agua para su saturación por un periodo de 12 a 24 horas, para después ser colocadas sobre un embudo que enviaría el agua emanada de la muestra directamente a una probeta graduada. Los centímetros cúbicos acumulados en la probeta se denominaron valores "Q" y se registraron cada 15 min. valor llamado "T". Con los datos obtenidos, se procedió a resolver la siguiente fórmula: $\mathrm{K}=\mathrm{Q} * \mathrm{~L} / \mathrm{H} * \mathrm{~T} * \mathrm{~A}$, donde "K" es el potencial hídrico del suelo.

\section{Medición de erosión por captura de sedimentos}

Para efectuar la medición del grado de erosión con este método, se emplearon dos áreas plantadas con piña bajo la siguientes condiciones: un sitio trabajado bajo escasa pendiente (lote 7305) versus un sitio trabajado bajo alta pendiente (lote 7312). En las dos áreas seleccionadas, se calculó con ayuda del Sistema de Posicionamiento Global (GPS) el porcentaje de la pendiente usado la siguiente fórmula: (Altura final - Altura inicial / Distancia recorrida) $* 100$.

En cada uno de los lotes se colocaron dos medios estañotes metálicos y se procedió a medir con el GPS el área de influencia de la cual se estima provendrían los sedimentos arrastrados por el agua de escorrentía. Se estableció un cronograma de monitoreo para realizar 6 mediciones de sedimentos una cada 6 días. En cada medición, se pesaría la cantidad de tierra húmeda retenida en los medios estañones con una balanza portátil y se tomaría una muestra de un kilo para ser secada en un horno convencional, con el fin de obtener sólo el valor de peso seco de suelo de ese kilo, que al multiplicarlo por la cantidad de kilos húmedo hallados en el medio estañón, daría el total de suelo perdido por erosión.

Cuando se concluye el proceso de medición, se realiza la sumatoria del suelo seco perdido por erosión, a cada contenedor por separado y se inicia el proceso de conversión de los datos como sigue: Cada área de influencia es convertida de metros cuadrados a hectáreas cuadradas (por ejemplo: $450 \mathrm{~m}^{2} \div 10,000 \mathrm{~m}^{2}=0,045 \mathrm{ha}$ ). La sumatoria de suelo seco de cada recipiente se extrapoló con regla de tres al valor en hectáreas obtenido anteriormente (Por ejemplo: si se obtuvieron $10 \mathrm{Kg}$. de peso seco, al aplicar la relación se obtiene: $10 \mathrm{~kg}$. suelo seco : 0,045 ha :: X kg. suelo seco : 1 ha. $X=222,2 \mathrm{Kg} / \mathrm{ha}$.)

La extrapolación del tiempo se logra de la siguiente manera: se multiplica el número de mediciones por el tiempo transcurrido entre cada una (6 mediciones una cada 6 días), generando un valor de 36 días totales del ensayo, además como en la zona de estudio la época lluviosa no es constante, si no que se presenta durante 8 meses al año y como la medición fue sólo en época lluviosa. se calcula cada mes con 30 días, que al ser multiplicados por los 8 meses, se obtienen 240 días bajo la presencia de lluvias que causa la erosión registrada. Con regla de tres se procede como sigue: $222,2 \mathrm{Kg} / \mathrm{ha}$ : 36 días :: X Kg/ha : 
240 días (influencia de lluvia al año) $X=1481,3$ $\mathrm{Kg} / \mathrm{ha} / \mathrm{año}$.

Con el dato convertido en kilogramos por hectárea por año, lo queda es transformar el valor de kilos a toneladas, simplemente dividiendo el dato entre mil kilogramos que equivalen a una tonelada. Continuado con el Ejemplo sería: $1481,3 \mathrm{~kg} / \mathrm{ha} / \mathrm{año} \div 1000 \mathrm{~kg} .=1,48 \mathrm{Ton} / \mathrm{ha} / \mathrm{año}$.

Todo el proceso de conversión se repitió para cada uno de los contenedores y al final los valores fueron promediados para obtener el valor estimado de erosión.

\section{Medición de erosión a través del análisis de pedestales}

La técnica consistió en medir en el campo las pequeñas columnas de tierra, que a pesar del impacto de la lluvia o el escurrimiento del agua, han logrado permanecer firmes bajo la protección de diversos objetos presentes en el terreno, como lo son pequeñas piedras, hojas o incluso retoños de vegetación.

En el campo, se ejecutó la medición de hasta 10 pedestales por hectárea y se procedió a desarrollar la siguiente fórmula para determinar la pérdida de suelo, en toneladas por hectárea por año: $\mathrm{P}=10000 * \mathrm{D}$ v * d.a. * f.t.

$\mathrm{P}=\quad$ pérdida de suelo en Ton/ha/año

$\mathrm{D} v=$ Distancia vertical (altura promedio de los pedestales en metros)

d. $a .=$ Densidad aparente del suelo en toneladas por metro cúbico

f. t. $=$ Factor tiempo equivalente al año.
Para determinar el factor tiempo, se utilizó como referencia el número de meses lluviosos que presenta el sitio al año y se dividió entre la cantidad de meses lluvioso que afectaron el área desde que se estableció el cultivo, hasta el momento de efectuar la medición de pedestales. Por ejemplo el año tiene 6 meses de lluvias y desde que se establece el cultivo hasta que se miden los pedestales, se contabilizaron 3 meses de lluvia, al efectuar la división el factor de tiempo sería 2.

\section{Resultados y discusión}

Medición de la pérdida de suelo por erosión hídrica

\section{Método de captura de sedimentos}

La medición de la erosión con este método, se realizó durante la época lluviosa, colocando 4 recipientes en los canales de desagüe dentro de las plantaciones de piña, de manera que se pudiese capturar las partículas de tierra arrastradas por el agua de escorrentía. Los dos primeros recipientes numerados como 1 y 2 fueron situados en el lote 7312 y los otros dos recolectores nombrados 3 y 4 se ubicaron en el lote 7305 . El cuadro 1 , resume las condiciones bajo las cuales cada recolector de sedimentos fue establecido.

$\mathrm{Al}$ término de las mediciones, se realizaron las correspondientes conversiones con los datos logrados hasta obtener el estimado de erosión para el terreno en estudio (Cuadro 2). Se aclarar, que en la desarrollo de esta experimentación se generaron problemas de rebalse de sedimentos en dos de los recolectores, por efecto de fuertes

CUADRo 1

Características de cada recolector de sedimentos en el campo

\begin{tabular}{lcccc}
\multicolumn{1}{c}{ Lotes No } & 7312 & & \multicolumn{2}{c}{7305} \\
Recolectores & 1 & 2 & 3 & 4 \\
Pendiente $\%$ & 22 y 18 & 15 & 2 & 3 \\
Área de influencia m & 2 & 770 & 2173 & 308 \\
Edad del cultivo meses & 7264 & 7 & 1 & 1 \\
Característica observadas & $\begin{array}{c}\text { Influencia de dos } \\
\text { pendientes, Diseño } \\
\text { siembre paralelo } \\
\text { a la pendiente }\end{array}$ & $\begin{array}{c}\text { Diseño siembre } \\
\text { paralelo a } \\
\text { la pendiente }\end{array}$ & $\begin{array}{c}\text { Terreno } \\
\text { escasamente } \\
\text { ondulado }\end{array}$ & $\begin{array}{c}\text { Terreno } \\
\text { escasamente } \\
\text { ondulado }\end{array}$ \\
\hline
\end{tabular}


Conversiones de los datos logrados en el ensayo

\begin{tabular}{|c|c|c|c|c|}
\hline Unidad de medida & $\begin{array}{c}\text { Recolector } \\
1\end{array}$ & $\begin{array}{c}\text { Recolector } \\
2\end{array}$ & $\begin{array}{c}\text { Recolector } \\
3\end{array}$ & $\begin{array}{c}\text { Recolector } \\
4\end{array}$ \\
\hline \multirow{6}{*}{$\begin{array}{l}\text { Kilogramos de suelo seco por } \\
\text { medición }\end{array}$} & 101.39 & 66.17 & 75.11 & 57.68 \\
\hline & 0.858 & 1.08 & & \\
\hline & 39.30 & 10.44 & & \\
\hline & 85.12 & 85.95 & 72.06 & 51.80 \\
\hline & 92.85 & 70.12 & 8.43 & 0.40 \\
\hline & 83.36 & 48.18 & 18.27 & 0.36 \\
\hline Total de kilogramos de suelo seco & 402.88 & 281.94 & 173.87 & 110.24 \\
\hline Metros cuadrados de influencia & $3,264.00$ & 770.00 & $2,173.00$ & 308.00 \\
\hline ha. cuadradas & 0.33 & 0.08 & 0.22 & 0.03 \\
\hline $\mathrm{Kg} / \mathrm{ha}$ & $1,234.31$ & $3,661.56$ & 800.14 & $3,579.22$ \\
\hline Kg/ha/año & $8,228.72$ & $24,410.39$ & $5,334.25$ & $23,861.47$ \\
\hline Ton/ha/año & 8.23 & 24.41 & 5.33 & 23.86 \\
\hline Promedio & \multicolumn{4}{|c|}{ 15.46 Ton/ha/año } \\
\hline
\end{tabular}

lluvias y el rápido flujo de agua en los canales de desagüe, lo que se reflujo visiblemente al momento de realizar los cálculos finales para determinar la cantidad de erosión que ocurre al año en el sitio.

Los resultados del ensayo, sin cuantificar el error sufrido a causa del desborde de sedimentos, arrojaron un valor promedio final que estimo la erosión para el área de estudio en 15 . Ton/ha/ año, pero tras la realización de un análisis lógico para averiguar la magnitud del error originado producto del desborde de sedimentos, se obtuvo un dato que estimó la erosión en alrededor de 48 Ton/ha/año (Cuadro 3). En la estimación de este dato, se hizo uso de los valores de erosión registrados para los recolectores tres y cuatro, debido a que estos no tuvieron pérdida de sedimentos, por lo que funcionaron como referencia para estimar el problema ocurrido en los otros dos recolectores.
El análisis procedió de la siguiente manera: en el recolector 3, el área de influencia es grande y el porcentaje de pendiente está cercano al $2 \%$, por lo que el valor de erosión obtenido ( 5 ton/ha/año) se considera aceptable. Para el caso del recolector 4, el área de influencia es mucho menor que en el tres, pero su pendiente es levemente mayor del 3\% y su efecto se puede notar en las 23 Ton/ ha/año medidas. En el caso del recolector 2, el área de influencia es sólo un poco mayor al doble que la del recolector 4 , pero en relación a la pendiente es tres veces mayor. En base a esta observación, se puede presumir que las condiciones influyentes en la erosión se duplican para el recolector 2 con respecto del recolector 4, por lo tanto el dato de $24 \mathrm{Ton} / \mathrm{ha} /$ año estimado al doble debería ser de 48 o más Ton/ha/año. Por ultimo en el recolector 1, el área de influencia es tres veces mayor que en el recolector 2 y tiene además la afectación de dos

CUADRO 3

Estimación de suelo perdido por erosión.

\begin{tabular}{ccccc} 
Recolectores & Área de influencia & Pendiente $\%$ & $\begin{array}{c}\text { Erosión registrada } \\
\text { Ton/ha/año }\end{array}$ & $\begin{array}{c}\text { Erosión supuesto } \\
\text { Ton/ha/año }\end{array}$ \\
$\mathbf{1}$ & 3,624 & 22 y 18 & 8.23 & 120 \\
$\mathbf{2}$ & 770 & 15 & 24.41 & 48 \\
$\mathbf{3}$ & 2,173 & 2 & 5.33 & 5 \\
$\mathbf{4}$ & 308 & 3 & 23.86 & 23 \\
Promedio & & & 15.46 Ton/ha/año & 49 Ton/ha/año \\
\hline
\end{tabular}


pendientes pronunciadas, por lo tanto el dato estimado se calcula que es dos y media veces más grande, que el calculado para el recolector 2, lo que arroja un valor de 120 Ton/ha/año. Con las estimaciones de los datos realizadas, sólo quedó promediar el valor, que como se mostró anteriormente ronda las 48 Ton/ha/año.

\section{Medición de la pérdida de suelo}

a través del análisis de pedestales

Para obtener un dato estimado sobre la pérdida de suelo con esta técnica, se procedió a realizar la medición de pedestales, en los dos sitios que se usaron en el método anterior, es decir el lote 7312 y el 7305, ejecutando dos secuencias de observación por cada uno (Cuadro 4). Cada secuencia de observación consta de por lo menos 8 mediciones de pedestales dentro de una misma hectárea.

Las dos secuencias de observación en cada lote se promediaron y los valores fueron manejados por separado, debido a que el lote 7305 poseía un tiempo de preparación distinto al primero, lo cual hizo necesario que se ejecutaran los cálculos de erosión por aparte en cada lote, para poder generar un promedio al final.

Se toman los valores de densidad aparente del suelo que fueron calculados en el área de estudio (Cuadro 5) y se promediaron, obteniendo el siguiente dato $0.978 \mathrm{~g} / \mathrm{cm}^{3}$, que se debió convertir a toneladas por metro cúbico, lo que da como resultado exactamente el mismo valor, pero en una unidad de medida más grande 0.978

CUADRO 4

Altura de pedestales medidos en el campo.

\begin{tabular}{|c|c|c|c|c|}
\hline \multirow{3}{*}{ Número de mediciones } & \multicolumn{4}{|c|}{ Secuencias de observación (altura en metros) } \\
\hline & \multicolumn{2}{|c|}{ Lote 7312} & \multicolumn{2}{|c|}{ Lote 7305} \\
\hline & № 1 & No 2 & № 1 & No 2 \\
\hline 1 & 0.005 & 0.011 & 0.005 & 0.008 \\
\hline 2 & 0.003 & 0.013 & 0.008 & 0.006 \\
\hline 3 & 0.013 & 0.013 & 0.008 & 0.009 \\
\hline 4 & 0.011 & 0.013 & 0.007 & 0.004 \\
\hline 5 & 0.012 & 0.011 & 0.003 & 0.006 \\
\hline 6 & 0.006 & 0.015 & 0.006 & 0.005 \\
\hline 7 & 0.005 & 0.009 & 0.007 & 0.007 \\
\hline 8 & 0.006 & 0.011 & 0.008 & 0.005 \\
\hline 9 & 0.013 & 0.009 & 0.010 & 0.006 \\
\hline 10 & 0.012 & & & \\
\hline 11 & 0.006 & & & \\
\hline Promedio (m) & 0.0084 & 0.0117 & 0.0069 & 0.0062 \\
\hline Promedio por lote (m) & \multicolumn{2}{|c|}{0.0101} & \multicolumn{2}{|c|}{0.0065} \\
\hline
\end{tabular}

\section{CUADRO 5}

Valores de densidad aparente calculados en el área de estudio.

\begin{tabular}{ccccccc} 
Muestra & P.C. & P.C. + S s. & P. S s. & V. C. & D. Ap. $/ \mathrm{cm}^{3}$ & Promedio $/ \mathrm{cm}^{3}$ \\
\hline 1 & 237.2 & 503.2 & 266 & 269.39 & 0.99 & \\
2 a & 237.2 & 528.1 & 290.9 & 269.39 & 1.08 & \\
2 b & 237.2 & 490.5 & 253.3 & 269.39 & 0.94 & 0.978 \\
3 a & 237.2 & 492.1 & 254.9 & 269.39 & 0.95 & \\
3 b & 237.2 & 487.2 & 250 & 269.39 & 0.93 & \\
\hline
\end{tabular}


Ton/ $\mathrm{m}^{3}$. Posteriormente, se determinó para cada lote un factor de tiempo, que en este caso correspondió al cociente de dividir los meses de época lluviosa al año que posee la zona, por los meses de época lluviosa que contiene el tiempo desde que se estableció la plantación de las áreas seleccionadas, hasta el momento de la medición de pedestales.

$\begin{array}{ll}\text { P. }= & \text { Peso (masa) } \\ \text { C. }= & \text { Cilindro } \\ \text { S s.= } & \text { Suelo seco } \\ \text { V.= } & \text { Volumen } \\ \text { D. Ap. }= & \text { Densidad Aparente } \\ \% .= & \text { Porcentaje }\end{array}$

Con base en lo anterior se obtuvo que, la zona posee 8 meses de época lluviosa (de octubre a enero y de mayo a agosto) y para el lote 7312, desde que se estableció la plantación (enero del 2007) hasta que se efectuó la medición de pedestales (septiembre 2007), los meses que contemplaron lluvia fueron 5, por lo que al realizar la división se logró como factor de tiempo 1,6. Para el lote 7305 se estimó el tiempo de igual forma, donde la plantación fue establecida en junio del 2007 y la medición de pedestales en septiembre del 2007, lo que genero un total de 4 meses de lluvia que, realizando la división da como factor de tiempo 2 para este último lote. En este punto se hace la aclaración de que la época lluviosa (mayo a agosto) se retrazó un mes, hecho que se refleja en los cálculos de meses con lluvia.

La estimación de la pérdida de suelo en cada lote, terminó con la aplicación de la siguiente fórmula: $\mathrm{P}=10000 * \mathrm{Dv} *$ d.a. $*$ f.t.

\section{Lote 7312}

$\mathrm{P}=10000 * 0,0101 * 0,978 * 1,6$

$\mathrm{P}=158,04$ ton/ha/año

\section{Lote 7305}

$\mathrm{P}=10000 * 0,0065 * 0,978 * 2$

$\mathrm{P}=127,14$ ton/ha/año

Con la premediación de los datos obtenidos en ambos lotes se estimó la erosión en 142.59 ton/ha/año.
Los datos de erosión calculados tanto con el primer método como con el este último, enmarcan la categoría de erosión dentro de un grado de severidad, según parámetros del MAG et al. (1994), que establecen que este tipo de erosión abarca los valores de suelo perdido que ronden de 50 a 200 ton/ha/año.

\section{Capacidad hídrica y densidad aparente como facilitadores de la erosión}

La zona de San Gerardo, presenta una asociación taxonómica de suelos principal de tipo Typic Humitropept (inceptisol típico de las zonas tropicales con presencia de humus) asociada con suelos Typic Dystropept (inceptisol típico del trópico con baja saturación de bases), que presentan problemas de fertilidad (Acón y asociados S.A., 1991).

En el área de estudio se efectuó un análisis sobre el estado de la conductividad hidráulica y la propiedad de densidad aparente superficial, que exhiben esto suelos inceptisoles dentro de la fincas, para establecer una relación que pudiera estar propiciando la pérdida de suelo por erosión hídrica.

Los resultados de las muestras de densidad aparente revelaron que, la densidad superficial es considerada como de categoría liviana, por alcanzar un valor promedio de $0.978 \mathrm{~g} / \mathrm{cm}^{3}$, valor que confirmaría su rápida conductividad hídrica con la muestra extraída a los $20 \mathrm{~cm}$, que registró un valor de $12.57 \mathrm{~cm} / \mathrm{h}$. Pero como un fuerte contraste al valor de capacidad hídrica superficial, la segunda muestra de conductividad tomada al nivel de $45 \mathrm{~cm}$, registró un dato de $0 \mathrm{~cm} / \mathrm{h}$. constituyéndose por defecto una capa de suelo poco permeable.

En resumen, la capa de suelo superficial (Fotografía 1), en las plantaciones, es altamente porosa y suelta, por lo cual se constituye en un factor que maximiza el potencial de la lluvia para lavar y remover tanto las partículas de tierra como los elementos agroquímicos adicionados al suelo, lo que es un problema que se agrava debido a la poca permeabilidad que muestra la capa de subsuelo, que tiene una constitución de 


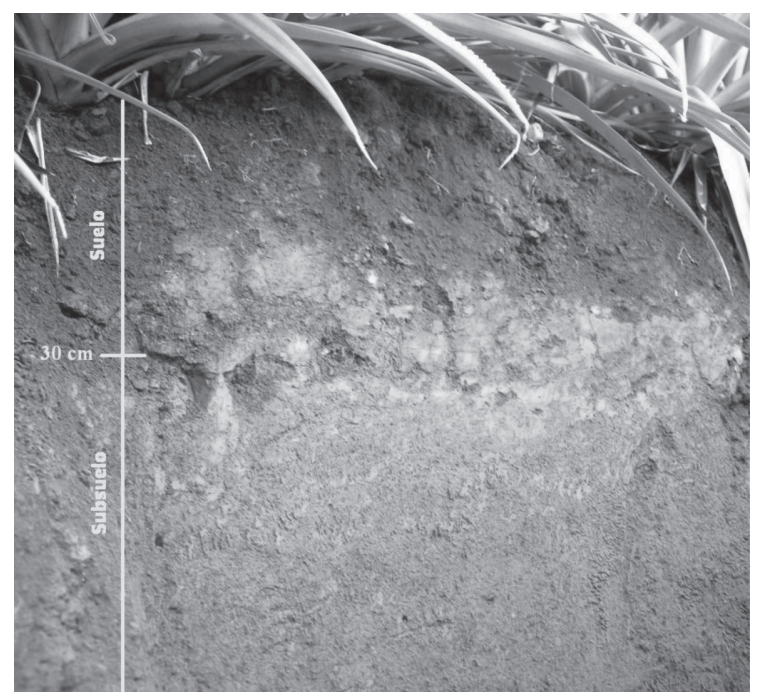

Fotografía 1. Capa de suelo y subsuelo en los cultivos de piña (M.Alvarez 1997)

materiales más finos y densamente enlazados que en la capa superior.

Además se puede hallar como responsable de las condiciones de que facilitan la erosión al intenso empleo de la rastras de discos para la preparación del suelo y la poca incorporación de material orgánico a la capa del subsuelo, que muestra un rápido asentamiento después de su preparación.

La alta mecanización que enfrenta los suelos de la actividad piñera en la zona estudiada, ha provocado fuerte problemas de sedimentación en los ríos (Fotografía 2) y arrastre de sustancias químicas a ellos (SUNII-MAG, 2006), cuyos efectos negativos se manifiestan en el dañando y muerte de mucha de la fauna y flora que depende del recurso líquido para subsistir.

\section{Conclusiones}

Con los datos obtenidos en la investigación realizada sobre los terrenos cultivados con piña, se concluye que, los altos niveles de erosión registrados se pudieron asociar de forma clara a los factores de capacidad hídrica y densidad aparente, por contribuir con la maximización del potencial de la lluvia para lavar las participas de tierra. Las condiciones de estos factores son en esencia producto de un intensivo uso de la rastra

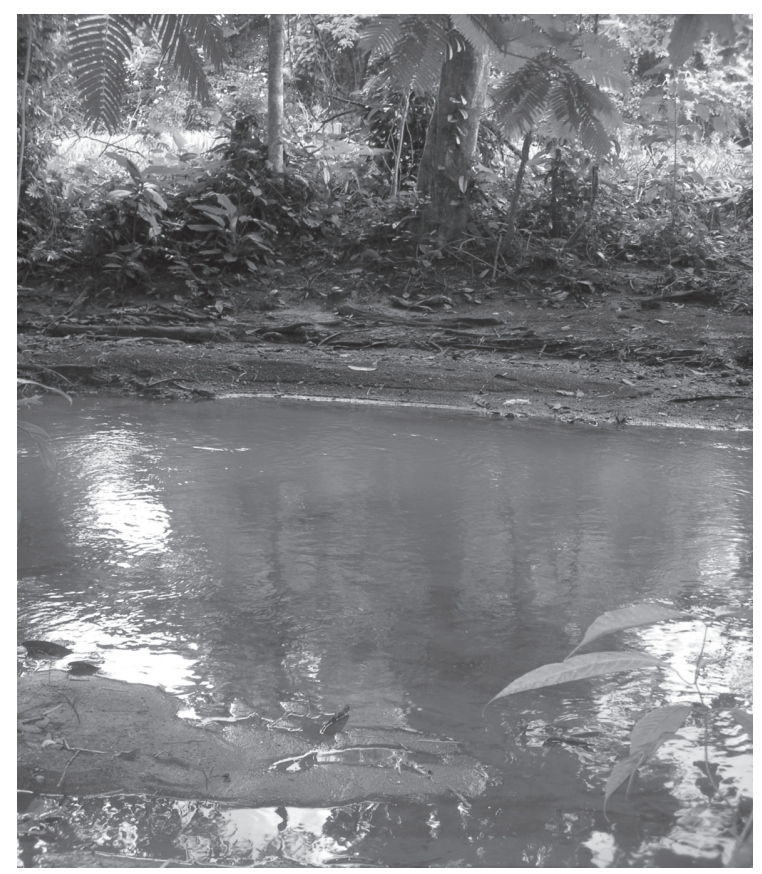

Fotografía 2. Quebrada colmatada con sedimentos, mostrando cultivos de piña al fondo. Tomada en la Quebrada Sonora (M.Alvarez 1997)

de discos que pulveriza excesivamente el suelo y la poca incorporación de material orgánico que permita mejorar las condiciones de estabilidad estructural de los agregados y aumente la precolación de las aguas a estratos más profundos. Es decir, al afectar la estructura de los suelos las condiciones de fragilidad del sistema edáfico es cada vez más acentuada, por lo que aumentan los procesos de erodabilidad y erosividad. Como recomendación se pretende mejorar este aspecto, favorecer la estructura o agregados del recurso, que se puede realizar mediante las buenas prácticas como la cobertura vegetal y la minimización del uso de maquinaria pesada.

\section{Referencias bibliográficas}

CADETI (Comisión Asesora sobre Degradación de Tierras, CR) - MINAE (Ministerio de Ambiente y Energía, CR). 1999. Primera jornada de sensibilización sobre degradación y desertificación de tierras. Memoria. San José, Costa Rica. 128 p.

MAG (Ministerio de Agricultura y Ganadería, CR) FAO (Organización de las Naciones Unidas para 
la Agricultura y la Alimentación, Roma); UNED (Universidad Estatal a distancia, CR). 1994. Manual de manejo y conservación de suelos y aguas. $2^{\text {a }}$ ed. EUNED. San José, Costa Rica. 300 p.

MAG; FAO. 1997. Extensión participativa para la agricultura conservacionista: conceptos, estrategias y metodologías. San José, Costa Rica. 49 p.

González, L. C. 2005. Expansión piñera en el Caribe. Opinión Ambiental. San José, Costa Rica. no. 29: 4-5.

IGNCR (Instituto Geográfico Nacional, CR). 1985. Hoja cartográfica "Río Cuarto 3347 II". Edición 2-IGNCR. San José, Costa Rica. Escala 1: 50 000. Color.
Programa Estado de la Nación. 2005. Programa Estado de la Nación en desarrollo humano Sostenible, Undécimo informe. San José, Costa Rica. p. 201 - 205.

Acón y Asociados S. A. 1991. Manual descriptivo de la leyenda del mapa de capacidad de uso de la tierra de Costa Rica escala 1:200. 000 (en línea). Consultado 28 de jul. del 2007. Formato pdf. Disponible en http:// www.mag.go.cr/bibliotecavirtual/manual-descriptivouso-tierra.pdf

SUNII (Sistema Unificado de Información Institucional, CR) / MAG. 2006. Programa de agencia de servicios agropecuarios 2003 - 2006 (La Virgen, Sarapiquí). Consultado 10 de Dic. del 2006. Formato pdf. Disponible en http:// www.mag.go.cr/regionales/p_ hn_virgen_03-06.pdf 
